\title{
FAST TARGET EXTRACTION BASED ON BAYESIAN BLOB ANALYSIS AND SIMULATED ANNEALING FOR UNDERWATER IMAGES
}

\author{
Wei Zhang, ${ }^{*}$ Ximeng Wang, ${ }^{*}$ Tao Chen, ${ }^{*}$ Lifeng Gao, ${ }^{*}$ Xixun Sun, ${ }^{*}$ and Hongliang Ren**
}

\begin{abstract}
As there are great absorption and scattering in water, it is difficult to extract the target region from an underwater image effectively. This paper investigated a Bayesian decision-making framework for segmenting underwater images, with the improved OTSU algorithm combined with the simulated annealing algorithm calculating the optimum threshold. The improved OTSU algorithm took fully into account grey values of pixels and their neighbours to have a better ability of filtering noise. The simulated annealing algorithm was contributed to reduce the amount of calculation and improved the efficiency of calculating the optimum threshold. Blob operators were used to exclude fake target regions based on Bayesian decisionmaking. The mathematical morphology operators were used to eliminate burrs and disturbances. The result of processing the images grabbed at pool experiments proved the better capability of segmentation with the proposed method.
\end{abstract}

\section{Key Words}

Segmentation, annealing algorithm, Blob analysis, Bayesian designmaking, mathematical morphology

\section{Introduction}

Image segmentation is a significant step of image processing, the image segmentation results will have a direct impact on the understanding of the image. According to the using feature, the classical segmentation methods can be classified into three kinds [1], [2]: (1) segmentation based on the bimodal histogram, (2) segmentation based on the edge and (3) segmentation based on the region.

The first method mainly includes global threshold segmentation, the advantages of this method are simple and fast. This method generally takes pixel grey value without

* College of Automation, Harbin Engineering University, People's Republic of China; e-mail: \{dawizw, 15603650167, chentao_, xiangkaikuaiche\}@163.com, 1198710835@qq.com

** Department of Biomedical Engineering, National University of Singapore, Singapore; e-mail: hlren@ ieee.org

Recommended by Dr. Peter Liu

(DOI: 10.2316/Journal.206.2017.2.206-4347) its spatial feature, so it is sensitive to the random disturbances, as it filters the noise but cannot obtain the segmentation image effectively when disturbances appeared, and it is hard to obtain the optimum threshold when the image is in low contrast [3], [4]. The second method detects the edge of region on the basis that the pixel grey value is not continuous in different regions so as to seldom characterize a region completely [5]. The difficulty of this method lies in the contradiction between anti-noise and accuracy of region detection. The third method puts the similar pixel grey value regions into segmentation regions. This method uses local spatial information. It can overcome the image segmentation of small space continuous shortcomings, but often causes excessive segmentation [6].

Because all the segmentation methods have defects, for different applications it needs different methods to extract ideal target regions. As the environment in deep ocean is very complex, as a result, targets and fake targets always mix together, and operators usually could not extract targets with only one segmentation method. To solve this problem, a new segmentation method combining improved planar OTSU algorithm, annealing algorithm, Blob analysis and mathematical morphology is proposed in this paper, as shown in Fig. 1.

\section{Threshold Based on Improved Planar OTSU}

Threshold segmentation has been widely applied with its simple and effective advantages. The OTSU algorithm has been widely applied because it is effective and adaptive. It is considered to be an optimal segmentation method of automatic threshold selection [7]-[9].

The underwater environment is quite complicated, the grey levels of targets and background are not different in obviously, meaning that there is no obvious double peak in the grey histogram. In this case, the original OTSU algorithm may miss the image information and even cause serious errors in segmentation. The planar OTSU algorithm takes not only grey values of pixels themselves but also the means grey values of their neighbours into account, so its capability of noise immunity has been 


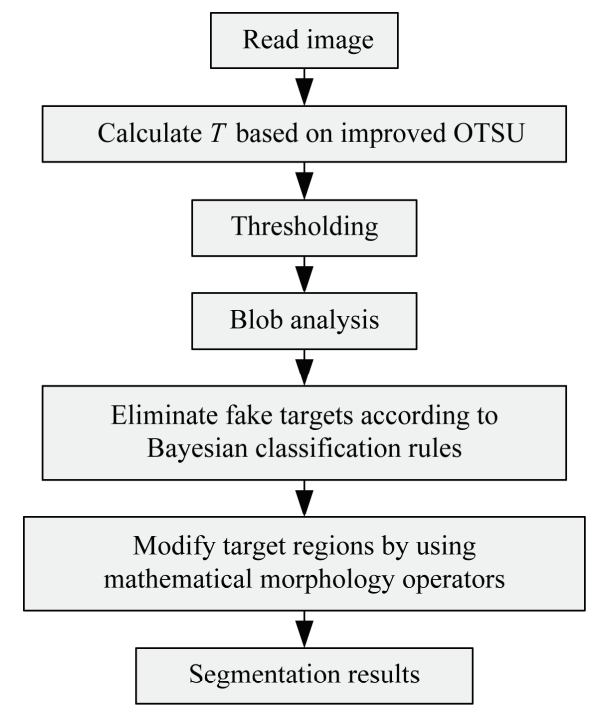

Figure 1. Flow chart of the method.

improved greatly compared to the original OTSU algorithm. The annealing algorithm is a kind of heuristic random searching method to solve global optimization problems by simulating the physical annealing process [10], compared with the traditional method of randomness, it has low computational complexity. This paper introduced the simulated annealing algorithm to speed up calculation of the improved planar OTSU.

\subsection{Planar OTSU Algorithm}

If $L$ be the grey level of the image, then the average grey level of its neighbour is also $L$. Let the frequency of pixel grey level $i$ is defined as:

$$
f_{i}=\sum_{j=0}^{L-1} f_{i j}
$$

Its probability is defined as:

$$
P_{i}=\sum_{j=0}^{L-1} P_{i j}
$$

Let the frequency of pixel grey level $i$ as:

$$
f_{j}=\sum_{i=0}^{L-1} f_{i j}
$$

Its probability is defined as:

$$
P_{j}=\sum_{i=0}^{L-1} P_{i j}
$$

The probability of targets and backgrounds are:

$$
\begin{gathered}
W_{t}=\sum_{i=0}^{s} P_{i} \\
W_{b}=\sum_{i=s+1}^{L-1} P_{i}
\end{gathered}
$$

The two corresponding average vectors are:

$$
\begin{gathered}
\mu_{t i}=\frac{\sum_{i=0}^{s} i P_{i}}{W_{t}} \\
\mu_{b i}=\frac{\sum_{i=s+1}^{L-1} i P_{i}}{W_{b}}
\end{gathered}
$$

The total average vector is:

$$
\mu_{i}=\sum_{i=0}^{L-1} \sum_{j=0}^{L-1} i P_{i j}=\sum_{i=0}^{L-1} i P_{i}
$$

Then, the one-dimensional variance among classes of $i$ is:

$$
\sigma_{B i}^{2}(s)=W_{t}\left(\mu_{t i}-\mu_{i}\right)^{2}+W_{b}\left(\mu_{b i}-\mu_{i}\right)^{2}
$$

the one-dimensional variance among classes of $j$ is:

$$
\sigma_{B j}^{2}(t)=W_{t}\left(\mu_{t j}-\mu_{j}\right)+W_{b}\left(\mu_{b j}-\mu_{j}\right)^{2}
$$

\subsection{Simulated Annealing Algorithm}

The simulated annealing algorithm is essentially a greedy algorithm, which in each iteration is generally selected a current optimum solution, but it introduced a random factor during the searching so can accept a bad solution to skip the trap of local optimum, and achieve global optimum solution.

Suppose that $f(x)$ is the optimization function, $g(x)$ is a state function, $R^{s}$ is feasible solution space, so simulated annealing algorithm is described below [11]:

(1) Choose one of the initial states $x_{0}, x_{0} \in R^{s}$, calculate the target function $f(x)$, choose the initial temperature $T_{0}$ and the length of Markov chain.

(2) Produce a random disturbance in $R^{s}$, set $x_{1}=g(x)$, then calculate the target function $f\left(x_{1}\right)$.

(3) If $f\left(x_{1}\right)<f\left(x_{0}\right)$, then accept $x_{1}$ as the current status, else judging whether accept $x_{1}$ according to Metropolis rules, if accept $x_{1}$ then set $x_{1}$ as the current status, else set $x_{0}$ as the current status.

(4) Based on a convergence criterion to judge whether to terminate the process of sampling, if yes, then go to (5), else go to (2).

(5) In accordance with cooling schemes decrease the control temperature $T$.

(6) Based on a convergence criterion to judge whether terminate the process of annealing, if yes, then go to (7), else go to (2).

(7) Output the current solution as optimum solution.

Set (10) and (11) separately as the optimization function, then introduce the simulated annealing algorithm to achieve the maximum. Set parameter of the simulated annealing algorithm, namely:

Cooling function:

$$
T_{k+1}=0.8 T_{k}
$$


Table 1

Kinds of Blob Operators

\begin{tabular}{|l|c|c|c|c|c|}
\hline Name & Expression & Range & $\begin{array}{c}\text { Influence of } \\
\text { occlusion }\end{array}$ & Inner holes & Circle \\
\hline Compactness & $C_{P}=\frac{L^{2}}{4 A \cdot \pi}$ & $C_{P} \geq 1$ & No & Sensitive & 1 \\
\hline Anisometry & $C_{A}=R_{a} / R_{b}$ & $C_{A} \geq 0$ & Yes & Non-sensitive & 1 \\
\hline Circularity & $C_{C}=\frac{A}{\pi \cdot \max ^{2}}$ & $C_{C} \in[0,1]$ & Yes & Non-sensitive & 1 \\
\hline Roundness & $\begin{array}{c}C_{R}=1-\frac{\sigma}{d}=\operatorname{sum}(\|p-q\|) / L \\
\sigma^{2}=\operatorname{sum}(\|p-q\|-d)^{2} / L\end{array}$ & $C_{R} \in[0,1]$ & Yes & Sensitive & 1 \\
\hline $\begin{array}{l}\text { Difference } \\
\text { deviation }\end{array}$ & $\begin{array}{c}d=\operatorname{sum}(\|p-q\|) / L \\
\sigma^{2}=\operatorname{sum}(\|p-q\|-d)^{2} / L\end{array}$ & $\sigma \geq 0$ & Yes & Sensitive & 0 \\
\hline Bulkiness & $C_{B}=\pi R_{a} \times R_{b} / A$ & $C_{B} \geq 1$ & No & Sensitive & 1 \\
\hline Convexity & $C_{V}=A / F_{C}$ & $C_{V} \in[0,1]$ & No & Sensitive & 1 \\
\hline
\end{tabular}

Initial temperature:

$$
T_{0}=1
$$

Ending temperature:

$$
T_{k}=1 e-8
$$

Status function:

$$
g(x)=1+\text { fix }(\text { rand } * 256)
$$

So, the optimal threshold is:

$$
\left(s^{*}, t^{*}\right)=\arg \max \left\{\sigma_{B i}^{2}(s), \sigma_{B j}^{2}(t)\right\}
$$

\subsection{The Threshold Segmentation Based on Im- proved OTSU Algorithm}

Compared with underwater images grabbed by a camera, the region of light source is smaller than that of background, and its proportion in the region of underwater image is small. The light source is the brightest and has the maximum grey value which is similar to the target's grey value, the location of the grey value of target light source is behind the maximum of the histogram. So the procedure of the threshold segmentation based on the improved OTSU algorithm is: first, acquire the maximum of the histogram and set its value to be $T_{0}$ from the underwater image. Then, put grey value of pixels which are greater than $T_{0}$ into the array, and the pixel grey value of target light source is increasing in proportion to the array. Finally, segment the underwater image with the threshold $L$ which is acquired by the OTSU algorithm calculated the array.

\subsection{Connected Regions Extraction}

In accordance with the above requested threshold $T$, we take global threshold segmentation of the image. Then we get the region the satisfying condition:

$$
\text { MinGrey } \leq f(x, y) \leq \text { MaxGrey }
$$

After threshold segmentation as the result of segmentation, it returns a region. Generally returning multiple objects in the region should be independent of each other. However, what we are interested in are closing shapes formed by several interconnected pixel sets. To get closing shapes, we have to calculate all the connected parts of the segmentation regions. The principle is to share a number for every connected region, and the number is the pixel value when output the image.

There are two definitions of connectivity: fourconnected region and eight-connected region. This paper adopts eight-connected region, because it is closer to people's true feeling.

\section{Eliminate Fake Targets}

The extraction of threshold segmentation may include fake targets. To extract real target regions accurately, we need to eliminate fake targets. Blob analysis could supply not only numbers, positions, shapes and orientations of regions but also topology of regions [12], [13]. Shapes of fake target regions are commonly irregular. So this paper describes connected regions using seven dimensions of Blob operators to eliminate fake target regions based on Bayesian decision-making rules.

\subsection{Blob Operators}

Definitions of seven different kinds of Blob operators are shown in Table 1. These operators are sensitive to circular 
and describe departure degree from different aspects. They could not describe too small regions because too small regions cannot form shapes. Each variable is defined as follows: $L$ is the length of a contour, $A$ is the area of a region, $R_{a}$ and $R_{b}$ are the major axis and minor axis of an ellipse separately, respectively, max is the farthest distance from the centre of a region to its contour, $p$ is an edge point in the contour, $q$ is the centre of a region and $F_{C}$ is the area of a bulge region.

\subsection{Eliminate Fake Target Regions Based on Bayesian Decision-Making Rules}

Let the prior probability of class $w_{i}$ be $p\left(w_{i}\right)$ and conditional probability density of the sample $x$ be $p\left(x \mid w_{i}\right)$. Posterior probability is $p\left(w_{i} \mid x\right)$. It means when $x$ is known, the conditional probability density of measured samples $w_{i}$ is $p\left(w_{i} \mid x\right)$. The scale factor $c$ is used to ensure the probability characteristics of $p\left(w_{i} \mid x\right)$. The Bayes formula is as follows:

$$
p\left(w_{i} \mid x\right)=\frac{p\left(x \mid w_{i}\right) p\left(w_{i}\right)}{c}
$$

Bayesian classification rules are: for the measurement $x$ of unknown sample, calculate its $p\left(w_{i} \mid x\right)$. For each $j \neq i$, if $p\left(w_{i} \mid x\right)$ is larger than $p\left(w_{j} \mid x\right)$, this condition belongs to category $i$. When $p\left(w_{0} \mid x\right)>p\left(w_{1} \mid x\right)$ it belongs to category 0 , otherwise it belongs to category 1. Inserting Bayes formula to (18) gives:

$$
p\left(x \mid w_{0}\right) p\left(w_{0}\right)>p\left(x \mid w_{1}\right) p\left(w_{1}\right)
$$

Namely:

$$
\frac{p\left(x \mid w_{0}\right)}{p\left(x \mid w_{1}\right)}>\frac{p\left(w_{1}\right)}{p\left(w_{0}\right)}
$$

The seven dimensions of Blob operators of regions obey Gaussian distribution approximately. It gets optimal parameter estimates by the average and the covariance of the sample. Calculating seven Blob operators of each sample, we obtain seven-dimensional described vector. Real targets belong to category 0 and fake ones belong to category 1 . The average and the variance matrix of the above two categories are $\boldsymbol{\mu}_{\mathbf{0}}, \boldsymbol{\mu}_{\mathbf{1}}, \mathbf{K}_{\mathbf{0}}$ and $\mathbf{K}_{\mathbf{1}}$. Then, the conditional probability density of seven-dimensional described vector is:

$$
p\left(x \mid w_{i}\right)=\frac{1}{(2 \pi)^{7 / 2}\left|\mathbf{K}_{\mathbf{i}}\right|^{1 / 2}} \exp \left[-\frac{1}{2} \frac{\left(x-\boldsymbol{\mu}_{\mathbf{i}}\right)^{T}\left(x-\boldsymbol{\mu}_{\mathbf{i}}\right)}{\mathbf{K}_{\mathbf{i}}}\right]
$$

The average and the variance of the sample are the optimal estimates under the maximum likelihood of Gaussian distribution, namely:

$$
\hat{\mu}_{i}=\frac{1}{n_{i}} \sum_{k=1}^{n_{i}} x_{i k}
$$

$$
\mathbf{K}_{\mathbf{i}}=\frac{1}{n_{i}} \sum_{k=1}^{n_{i}}\left(x_{i k}-\hat{\mu}_{i}\right)\left(x_{i k}-\hat{\mu}_{i}\right)^{T}
$$

Inserting (21) to (20), it gets discriminant function under Gaussian conditional probability:

$$
\begin{array}{r}
\ln \frac{\left|\mathbf{K}_{\mathbf{0}}\right|}{\left|\mathbf{K}_{\mathbf{1}}\right|}+\left(x-\boldsymbol{\mu}_{\mathbf{0}}\right)^{T} \mathbf{K}_{\mathbf{0}}^{-1}\left(x-\boldsymbol{\mu}_{\mathbf{0}}\right) \\
-\left(x-\boldsymbol{\mu}_{\mathbf{1}}\right)^{T} \mathbf{K}_{\mathbf{1}}^{-1}\left(x-\boldsymbol{\mu}_{\mathbf{1}}\right)<T^{*}
\end{array}
$$

Where discriminant threshold $T^{*}=-2 \ln \left[p\left(w_{1}\right) / p\left(w_{0}\right)\right]$ belongs to category 0 when $(24)$ is tenable. Otherwise, it belongs to category 1 .

\section{Modify Target Regions}

Mathematical morphology is the analysis of geometry and structure of mathematical methods. It is established on the basis of geometric algebra, using the set theory method to quantitative description of geometric structure [14], [15]. The basic operation of mathematical morphology includes the erosion, the dilation, the opening and closing operation.

Erosion, as it is known, will corrode the boundary of the target, and the result of the erosion is in relation with itself and selected structure elements. If target is smaller than the selected structure elements of erosion, then the erosion will decrease the target region by one lap, else the target region will be lost. If only a part of the target region is smaller than the selected structure elements, the erosion will disconnect the narrow connection. It can be described as $A$ and $S$ are sets of elements in the $S$, corrode $A$ with $S$, which can be defined by:

$$
A \Theta S=\left\{z \mid(S)_{z} \subseteq A\right\}
$$

On the contrary to erosion, dilation will make the boundary of target larger. The result of the dilation is in relation with itself and selected structure elements. Dilation usually is used to connect the slot of the same target which is disconnection in the image. It can be can be defined by:

$$
A \oplus S=\left\{z \mid(\widehat{S})_{z} \cap A \neq \varnothing\right\}
$$

Open operation includes taking erosion at first and then dilation, and it usually processes the boundary of image to be more smooth and wipes off burrs. Compared to erosion, open operation not only filters noise but also improves the effect of the boundary of target. It can be defined by:

$$
A \circ S=(A \Theta S) \oplus S
$$

Closed operation includes taking dilation at first and then erosion, it can processes the boundary of image to be more smooth, fills interruptions and floats holes [16], [17]. It can be defined by:

$$
A \bullet S=(A \oplus S) \Theta S
$$




\subsection{Correction of Target Regions}

Many burrs and disturbance regions still exist after the anterior two steps, so it is hard to find the real edge of the target regions. This paper adopts mathematical morphology operators to wipe off burrs and disturbances and regularize shapes of target regions.

First, corrode the regions. Structuring element should be larger than the disturbance region but smaller than the target region. But this method will make the size of the target region smaller than the real region. To solve this problem, this paper takes dilation structuring element that is the same as the erosion one because the erosion and the dilation are inverse operation to each other. Second, take opening operation. The structuring element should be larger than the maximum burns and smaller than the target region. This method can disconnect the intermittent of burns and wipe off protuberances. As a result, it makes contour line smooth. The opening operation could make the edges of region concave and produce some long thin

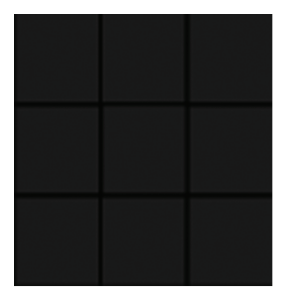

(a)

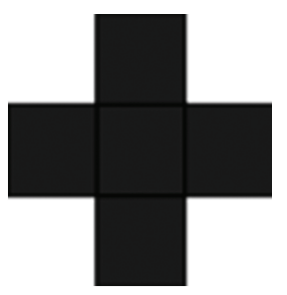

(b)
Figure 2. (a) $B_{4}$ and (b) $B_{8}$.

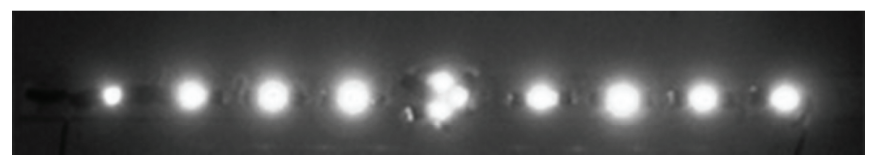

Figure 3. The light system. groove. Therefore, it needs the closing operation. Using the same structuring element as the opening one to make up concave long thin grooves, we get solid polygon regions. This method does not change the size of target regions.

After mathematical morphology management, burns and disturbance can be wiped off. But the shape of targets region is irregular. Generally, the shape of targets region is regular by designing. Therefore, the region is not ideal and will make it hard to extract features. To regularize region edge, it needs to track all the points of region edges and connect these points into a regular contour line.

To farthest approximate real region edge, we need to select suitable structuring element $B$. There are two kinds of edges: four-connected edge and eight-connected edge. As it is shown in Fig. 2 eight-connected edge is more exquisite. Therefore, this paper adopts eight-connected structuring element $B_{8}$.

\section{Experiment Results}

The validity of the proposed method was assessed by processing the images grabbed during the pool experiment. The target of this trial was a light system fixed at the bottom of a dock. The light system was composed of nine lights fixed along the longitudinal mid line of the dock, where the heart-shaped light was in the middle, and the other eight circle lights laid two sides of the heart-shaped light, as shown in Fig. 3.

In the experiment, it can be found out that the distance between each light is closer, the image's effect is better, the image contrast is higher, the light source region is more ideal with less disturbances by the fake light source, there is nearly no disturbance by the fake light source in $2 \mathrm{~m}$. On the contrary, the distance is longer, the light of the background of image is stronger, the probability of light source region is less ideal and that of the fake light source is increasing. The light region is preliminary extracted and discriminated in guiding image between 2 and $4 \mathrm{~m}$, as shown in Fig. 4(a)-(c).

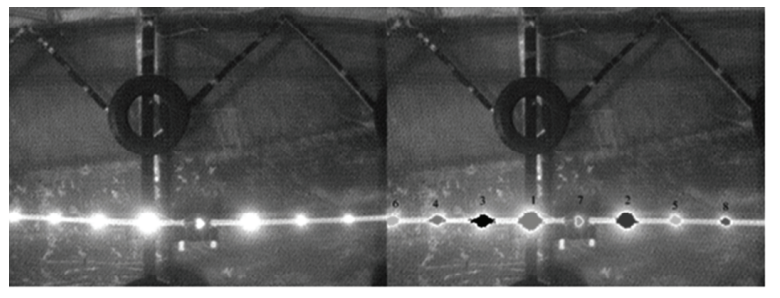

(a)

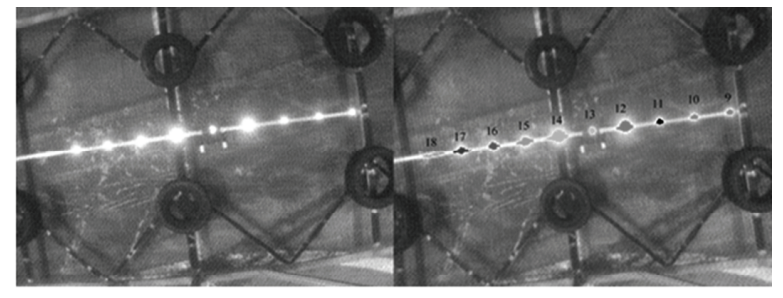

(b)

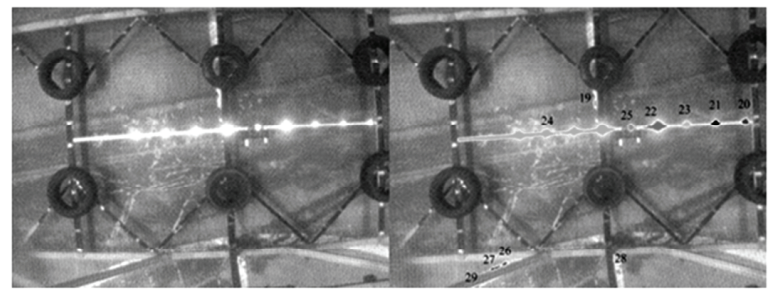

(c)

Figure 4. A series of images that each left one of (a), (b), (c) is the original image, and the others are results of thresholding: (a) taken nearly $2.5 \mathrm{~m}$; (b) taken nearly $3.5 \mathrm{~m}$; and (c) taken nearly $4 \mathrm{~m}$. 
Table 2

Results of Blob Analysis

\begin{tabular}{|c|c|c|c|c|c|c|c|c|}
\hline $\begin{array}{l}\text { Region } \\
\text { number }\end{array}$ & $C_{P}$ & $C_{A}$ & $C_{C}$ & $C_{R}$ & $\sigma$ & $C_{B}$ & $C_{V}$ & Class \\
\hline 1 & 1.846 & 1.531 & 0.461 & 0.786 & 2.121 & 1.051 & 0.85 & 0 \\
\hline 2 & 4.533 & 1.462 & 0.365 & 0.786 & 4.615 & 1.074 & 0.799 & 0 \\
\hline 3 & 4.271 & 2.178 & 0.222 & 0.636 & 4.539 & 1.151 & 0.7 & 0 \\
\hline 4 & 3.207 & 1.278 & 0.482 & 0.845 & 2.649 & 1.045 & 0.817 & 0 \\
\hline 5 & 3.955 & 2.009 & 0.267 & 0.694 & 4.996 & 1.118 & 0.747 & 0 \\
\hline 6 & 1.662 & 1.265 & 0.678 & 0.851 & 1.299 & 1.041 & 0.867 & 0 \\
\hline 7 & 1.912 & 1.417 & 0.462 & 0.79 & 1.568 & 1.06 & 0.836 & 0 \\
\hline 8 & 1.414 & 1.312 & 0.669 & 0.803 & 1.326 & 1.063 & 0.922 & 0 \\
\hline 9 & 1.207 & 1.089 & 0.793 & 0.751 & 0.692 & 1.028 & 0.864 & 0 \\
\hline 10 & 1.486 & 1.395 & 0.932 & 0.782 & 1.082 & 1.012 & 0.835 & 0 \\
\hline 11 & 1.536 & 1.362 & 0.606 & 0.748 & 0.813 & 1.134 & 0.831 & 0 \\
\hline 12 & 1.448 & 1.33 & 0.767 & 0.73 & 2.609 & 1.075 & 0.872 & 0 \\
\hline 13 & 1.352 & 1.347 & 0.736 & 0.706 & 2.394 & 1.078 & 0.841 & 0 \\
\hline 14 & 2.219 & 1.983 & 0.436 & 0.624 & 3.392 & 1.114 & 0.78 & 0 \\
\hline 15 & 2.089 & 1.517 & 1.313 & 0.679 & 1.658 & 1.045 & 0.761 & 0 \\
\hline 16 & 1.136 & 1.167 & 0.831 & 0.824 & 3.128 & 1.022 & 0.904 & 0 \\
\hline 17 & 2.249 & 2.002 & 0.247 & 0.661 & 4.56 & 1.155 & 0.798 & 0 \\
\hline 18 & 7.759 & 2.614 & 0.149 & 0.407 & 2.268 & 2.685 & 0.413 & 1 \\
\hline 19 & 3.091 & 3.172 & 0.331 & 0.367 & 1.581 & 1.454 & 0.614 & 1 \\
\hline 20 & 1.107 & 1.098 & 0.933 & 0.841 & 0.662 & 1.029 & 0.904 & 0 \\
\hline 21 & 1.653 & 1.586 & 0.452 & 0.737 & 1.412 & 1.078 & 0.839 & 0 \\
\hline 22 & 3.226 & 2.088 & 0.202 & 0.624 & 4.968 & 1.19 & 0.739 & 0 \\
\hline 23 & 1.672 & 1.429 & 0.523 & 0.771 & 1.311 & 1.083 & 0.823 & 0 \\
\hline 24 & 22.305 & 26.562 & 0.026 & 0.412 & 48.991 & 1.558 & 0.42 & 1 \\
\hline 25 & 1.092 & 1.203 & 0.806 & 0.864 & 0.675 & 1.024 & 0.942 & 0 \\
\hline 26 & 1.138 & 1.764 & 0.712 & 0.65 & 0.859 & 1.033 & 0.905 & 0 \\
\hline 27 & 3.036 & 6.136 & 0.192 & 0.344 & 2.661 & 1.143 & 0.733 & 1 \\
\hline 28 & 3.398 & 1.196 & 0.481 & 0.499 & 1.57 & 1.504 & 0.633 & 1 \\
\hline 29 & 1.789 & 1.617 & 0.698 & 0.655 & 1.11 & 1.131 & 0.814 & 0 \\
\hline
\end{tabular}

Due to underwater light colours, grey values of light source in grey image are also different. The grey value of white light source is 255. It is the maximum. Therefore, this paper regards $T$ from the improved OTSU algorithm as MinGrey and MaxGrey is 255. The results of threshold segmentation are shown in Fig. 4. It detects 29 light regions and numbering them one by one.

Table 2 shows the results of Blob analysis and classification.
Figure 4(a) is taken nearly $2.5 \mathrm{~m}$, which has a rather ideal effect. Extract eight preliminary regions with threshold segmentation, label eight high brightness regions with 1-8, which are true and judged as actual light sources. Figure 4(b) is taken nearly $3.5 \mathrm{~m}$, is father than Fig. 4(a), the general brightness increases, a fake light source appears among the 10 regions of threshold segmentation, so number 18 at the left end of light source is correctly discriminated in class 1 , while the other 9 light sources is correctly 


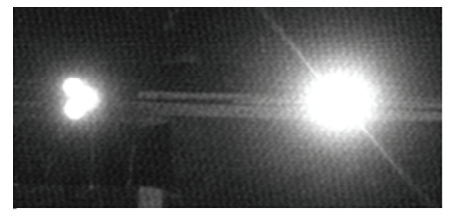

(a)

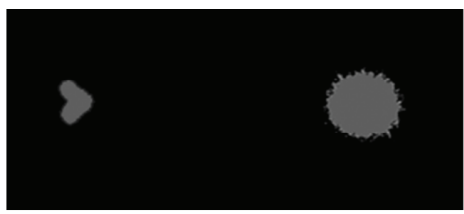

(b)

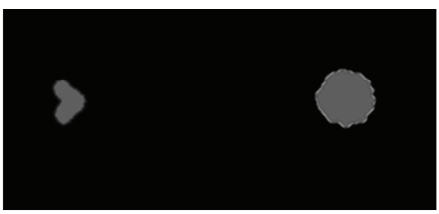

(c)

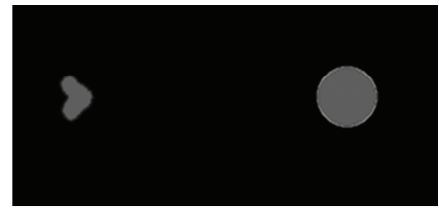

(d)

Figure 5. A series of images that the process of obtaining results of modifying light regions: (a) original image; (b) result of thresholding; (c) after basic operator; and (d) after regularization.

discriminated in class 0 . Figure $4(\mathrm{c})$ is taken nearly $4 \mathrm{~m}$, because the light of background is pretty strong, the reflection interference is rather serious. Only 5 of 14 regions of threshold segmentation are actual light sources. Number 24 is the fake light source which is composed of reflection of 4 light sources, other light sources such as numbers 19 and 26-29 are high brightness regions at the bottom of the pool. The discrimination result is 5 actual light sources are correctly judged, and 4 of 6 fake light sources are correctly judged, number 26 and 29 are misjudged as actual light sources.

To observe the effect of shape correcting clearly, we amplified heart-shaped light regions. The results are shown in Fig. 5, where (a) is the original image, (b) is the image after thresholding, first corrode the circle with radius of $3.5 \mathrm{~m}$ with structure element in Fig. 5(b), then take a dilation to this structure element $B$, finally, take the open and closed operation to the circle of which radius is $5.5 \mathrm{~m}$ with the structure element $B,(\mathrm{c})$ is the image after basic operators of mathematical morphology, (d) was the image after regularization. From Fig. 5, we can see that burrs and disturbance regions had been wiped off after using mathematical morphology operators, so it is feasible to conduct other operations, like character extraction, continually.

\section{Conclusion}

As we all know that the environment in deep ocean is very complex, as a result, targets and fake targets always mix together, and operators usually could not extract targets using only one segmentation method. To solve this problem, this paper adopts a new segmentation method combining improved planar OTSU algorithm, annealing algorithm, Blob analysis and mathematical morphology. It deals with the images grabbed during the pool experiments using the above methods. The results of the experiments proved the accuracy and efficiency of the method and acquired the real target regions. The deficiency of the proposed method is that high brightness regions at the bottom of the pool are misjudged as actual light sources because of reflection, so the future work is considering and eliminating the impact of environment of water, for example, the disturbance of impurities, scattering, reflection and so on.

\section{Acknowledgements}

This research work was supported by the Youth Project of National Natural Science Foundation of China (51309067/ E091002), the Science Foundation of Heilongjiang Province (E2016020), the Fundamental Research Funds for the Central Universities (HEUCF160407), the China Postdoctoral
Science Foundation funded project (2015M571391) and the Postdoctoral foundation of Heilongjiang Province (LBHZ15042).

\section{References}

[1] M. Sonka, V. Hlavac, and R. Boyle, Image processing, analysis, and machine vision (Beijing: People's Posts and Telecommunications Publishing House, 2003).

[2] C. Steger, M. Ulrich, and C. Wiedemann, Machine vision algorithms and applications (Beijing: Tsinghua University Press, 2008).

[3] M. Zhang, X. Li, and Y. Wang, Underwater color image segmentation based on weight adjustment for color-togray, Journal of Harbin Engineering University, 36(5), 2015, 707-713.

[4] M.Y. Choong, W.Y. Kow, Y.K. Chin, et al., Image segmentation via normalised cuts and clustering algorithm, IEEE Int. Conf. Control System, Penang, Malaysia, 2012, 430-435.

[5] J. Chen, J. Li, D. Pan, et al., Edge-guided multiscale segmentation of satellite multispectral imagery, IEEE Transactions on Geoscience and Remote Sensing, 50(11), 2012, 4513-4520.

[6] H. Yang and X. Zhou, Comparison of image segmentation methods, Computer Knowledge and Technology, 5(9), 2009, 2440-2441.

[7] M. Hu, M. Li, and R. Wang, Application of an improved OTSU algorithm in image segmentation, Journal of Electronic Measurement and Instrument, 24(5), 2010, 443-449.

[8] C. Xu, G. Peng, and H. Zhang, Fast algorithm for 2D OTSU thresholding algorithm, Journal of Computer Applications, 32(5), 2012, 1258-1260.

[9] L. Wan, H. Bai, and J. Dai, Extended optimal OTSU thresholding method of image segmentation, Journal of Harbin Engineering University, 24(3), 2003: 326-329.

[10] X. Li, H. Liu, and L. Chang, Multi-particle swarm co-evolution algorithm based on simulated annealing method, Application Research of Computers, 26(1), 2009, 71-74.

[11] P. Deng, Z. Fang, and S. Xiong, Noise adaptive filter based on SA, Electronic Design Engineering, 19(10), 2011, 132-135.

[12] G. Hu, Efficient Blob analysis of binary image for defects inspection in optical films, Journal of Computer Applications, 31(10), 2011, 2767-2769.

[13] D. duc Nguyen, T.C. Pham, X.D. Pham, et al., Finger extraction from scene with grayscale morphology and BLOB analysis, IEEE Int. Conf. Robotics and Biomimetics, Bangkok, 2008, 324-329. doi: 10.1109/ROBIO.2009.4913024.

[14] S. Sarah, Blob analysis and edge detection in the real world, Evaluation Engineering, 45(8), 2006, 46-49.

[15] Y. Zhu, J. Kan, J. Liu, et al., Application of mathematical morphology for image segmentation in waste wood based panel connectors detecting system, Int. Conf. Information, Networking and Automation (ICINA), Kunming, 2010, pp. V1-14-V1-17.

[16] K. Ye and M. Zhan, Image segmentation algorithm based on mathematical morphology and active edgeless contour model without edges, Journal of Computer Applications, 29(9), 2009, 2398-2401.

[17] R.C. Gonzalez and R.E. Woods, Digital image processing (Beijing: Electronic Industry Press, 2010). 


\section{Biographies}
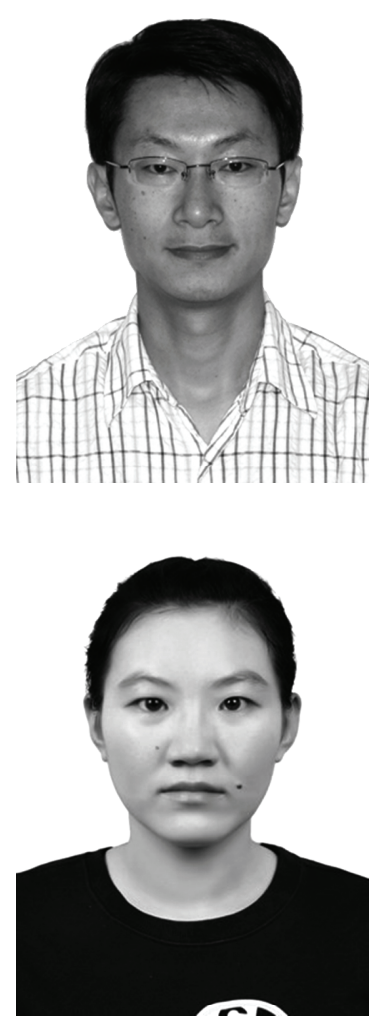

Ximeng Wang was born in Heilongjiang, China, and received her B.Sc. degree in Automation in 2016 from Harbin University of Science and Technology. She is now an M.S. student with the Laboratory of Unmanned Underwater Vehicle, under the supervision of Professor Wei Zhang. Her current research interests include image processing of underwater.

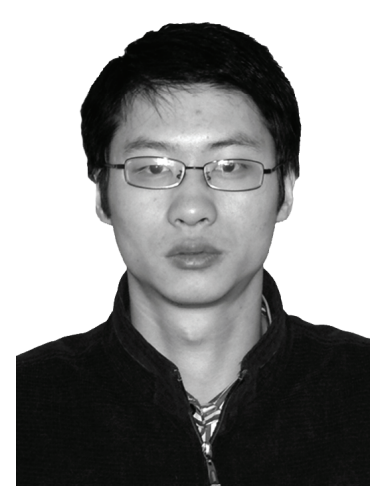

Tao Chen was born in Henan, China, and received his Ph.D. degree in Control Theory and Control Engineering in 2011 from Harbin Engineering University. He is currently a professor in Harbin Engineering University. His research interests include UUV route planning and unmanned intelligence control.

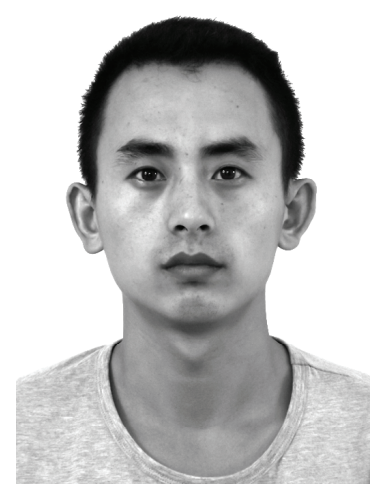

Lifeng Gao was born in Heilongjiang, China, and received his B.Sc. degree in automation in 2012 from Heilongjiang Institute of Science and Technology. He is now an M.S. student with the Laboratory of Unmanned Underwater Vehicle, under the supervision of Professor Wei Zhang. His current research interests include object tracking and image processing.

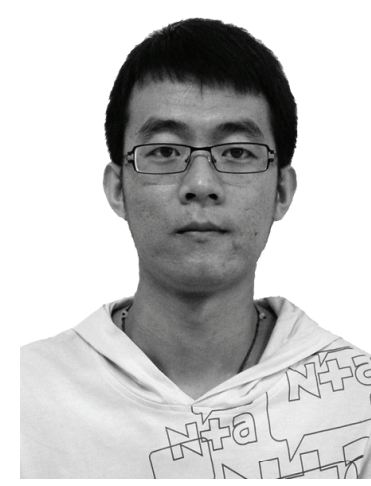

Xixun Sun was born in Hebei, China, and received his B.Sc. degree in Electrical Engineering and Automation in 2014 from Heilongjiang University. He is now an M.S. student with the Laboratory of Unmanned Underwater Vehicle, under the supervision of Professor Wei Zhang. His current research includes interests tracking control of underwater vehicle.

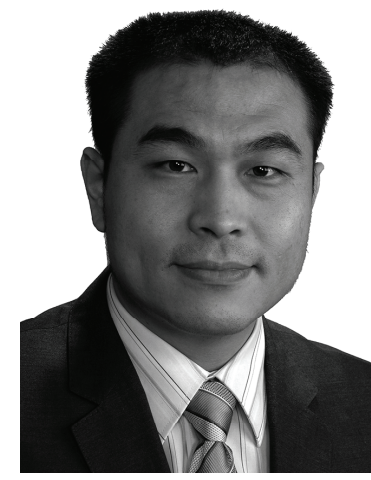

Hongliang Ren is currently an assistant professor in the Biomedical Engineering Department of National University of Singapore (NUS). He received his Ph.D. in Electronic Engineering (specialized in Biomedical Engineering) from the Chinese University of Hong Kong (CUHK) in 2008. His main areas of interest include image guidance, biomedical mechatronics, robotics and sensing. 